\title{
Tunneling current in triplet $f$-wave superconductors with horizontal lines of nodes
}

\author{
N. Stefanakis \\ Department of Physics, University of Crete, P.O. Box 2208, GR-71003, Heraklion, Crete, Greece
}

(November 5, 2018)

\begin{abstract}
We calculate the tunneling conductance spectra of a normalmetal/insulator/triplet superconductor using the Blonder-Tinkham-Klapwijk (BTK) formulation. Possible states for the superconductor are considered with horizontal lines of nodes, breaking the time reversal symmetry. These results would be useful to discriminate between pairing states in superonductor $\mathrm{Sr}_{2} \mathrm{RuO}_{4}$ and also in $\mathrm{UPt}_{3}$.

74.20. z, 74.50.+r, 74.80.Fp
\end{abstract}

Typeset using REVTEX 


\section{Introduction}

The resent discovery of superconductivity in $\mathrm{Sr}_{2} \mathrm{RuO}_{4}$ has attracted much theoretical and experimental interest [1]. Knight-shift measurements show no change when passing through the superconducting state and is a clear evidence for spin triplet pairing state [2]. Muon spin rotation experiments show that the time reversal symmetry is broken for the superconductor $\mathrm{Sr}_{2} \mathrm{RuO}_{4}[3]$. The linear temperature dependence of the nuclear spin lattice relaxation rate $1 / T_{1}$ of ${ }^{101} \mathrm{Ru}$ bellow $0.4 K$ [4], and specific heat measurements [5] are consistent with the presence of line nodes within the gap as in the high $T_{c}$ cuprate superconductors.

Knight-shift measurements show that the parity of the pairing function of $\mathrm{UPt}_{3}$ is odd and a spin triplet pairing state is realized [6]. Muon spin rotation experiments show that the time reversal symmetry is broken bellow $T_{c 2}$ for the superconductor $\mathrm{UPt}_{3}$ [7]. The nuclear spin lattice relaxation rate $1 / T_{1}$ and specific heat measurements are consistent with the presence of line nodes within the gap as in the high $T_{c}$ cuprate superconductors.

In tunneling experiments involving singlet superconductors both line nodes and time reversal symmetry breaking can be detected from the V-like shape of the spectra, and the splitting of the zero energy conductance peak (ZEP) at low temperatures respectively [8 11]. Electron tunneling in $\mathrm{Sr}_{2} \mathrm{RuO}_{4}$ has been studied in Refs. [12 114 for spin triplet pairing states with vertical lines of nodes. Electron tunneling in $\mathrm{UPt}_{3}$ has been studied in Ref. 15.

In this paper we discuss the tunneling effect in normal-metal/triplet superconductor with horizontal line nodes taking into account three dimensional effects. For the triplet superconductor $\mathrm{Sr}_{2} \mathrm{RuO}_{4}$ we shall assume three possible pairing states of three dimensional order parameter, having horizontal lines of nodes, which run parallel to the basal plane and break the time reversal symmetry. The first two are the $f$-wave states proposed by Hasegawa et al., [16] having $A_{1 g} \times E_{u}$ symmetry. The other one is the $f$-wave pairing symmetry proposed by H. Won and K. Maki [17].

For the triplet superconductor $\mathrm{UPt}_{3}$ we shall assume two possible pairing states of three dimensional order parameter, having horizontal lines of nodes, which run parallel to the basal plane and break the time reversal symmetry. These are the planar and bipolar pairing 
states proposed by Machida et al. 18.

\section{Theory for the tunneling conductance}

The interface has a $\delta$-functional form is perpendicular to the $z$-axis and is located at $z=0$ as seen in Fig. 11(a) (xy-interface). Alternatively we consider the situation where the interface is perpendicular to the $x$-axis and is located at $x=0$ (zy-interface), (see Fig. 1(b)). We assume a semi-infinite double layer structure and a spherical Fermi surface. The motion of quasiparticles in inhomogeneous superconductors is described by the solution of the Bogoliubov-deGennes(BdG) equations. The effective pair potential is given by

$$
\Delta_{\rho \rho^{\prime}}(\mathbf{k}, \mathbf{r})=\Delta_{\rho \rho^{\prime}}(\phi, \theta) \Theta(z)[\Theta(x)]
$$

for the $x y[z y]$-interface, where $k_{x}, k_{y}, k_{z}=\cos \phi \sin \theta, \sin \phi \sin \theta, \cos \theta . \quad \phi$ is the azimuthal angle in the $x y$-plane, $\theta$ is the polar angle. The quantities $\rho, \rho^{\prime}$ denote spin indices.

Suppose that an electron is injected from the normal metal with momentum $k_{x}, k_{y}, k_{z}$, and the interface is perpendicular to the $z$-axis. The electron (hole) like quasiparticle will experience different pair potentials $\Delta_{\rho \rho^{\prime}}(\phi, \theta)\left(\Delta_{\rho \rho^{\prime}}(\phi, \pi-\theta)\right)$. When the interface is perpendicular to the $x$-axis, the electron (hole) like quasiparticle will experience different pair potentials $\Delta_{\rho \rho^{\prime}}(\phi, \theta)\left(\Delta_{\rho \rho^{\prime}}(\pi-\phi, \theta)\right)$. The coefficients of the Andreev and normal reflection for the $x y$-interface are obtained by solving the BdG equations under the following boundary conditions

$$
\begin{gathered}
\left.\Psi(\mathbf{r})\right|_{z=0_{-}}=\left.\Psi(\mathbf{r})\right|_{z=0_{+}} \\
\left.\frac{d \Psi(\mathbf{r})}{d z}\right|_{z=0_{-}}=\left.\frac{d \Psi(\mathbf{r})}{d z}\right|_{z=0_{+}}-\left.\frac{2 m V}{\hbar^{2}} \Psi(\mathbf{r})\right|_{z=0_{-}}
\end{gathered}
$$

while for the $z y$ interface the boundary conditions are

$$
\begin{gathered}
\left.\Psi(\mathbf{r})\right|_{x=0_{-}}=\left.\Psi(\mathbf{r})\right|_{x=0_{+}} \\
\left.\frac{d \Psi(\mathbf{r})}{d x}\right|_{x=0_{-}}=\left.\frac{d \Psi(\mathbf{r})}{d x}\right|_{x=0_{+}}-\left.\frac{2 m V}{\hbar^{2}} \Psi(\mathbf{r})\right|_{x=0_{-}}
\end{gathered}
$$


Using the obtained coefficients the tunneling conductance for the $x y$ interface is calculated using the formula $\left.\sigma_{(} E\right)=\sigma_{\uparrow}(E)+\sigma_{\downarrow}(E)$, where the conductance for spin-up(-down) quasiparticle is given by the relation

$$
\sigma_{\uparrow[\downarrow]}(E)=\frac{\int_{0}^{2 \pi} \int_{0}^{\frac{\pi}{2}} \bar{\sigma}_{\uparrow[\downarrow]}(E, \phi, \theta) \sin \theta \cos \theta d \phi d \theta}{\int_{0}^{2 \pi} \int_{0}^{\frac{\pi}{2}} 2 \sigma_{N} \sin \theta \cos \theta d \phi d \theta},
$$

where the normal-state conductance is given by

$$
\sigma_{N}=\frac{\cos ^{2} \theta}{\cos ^{2} \theta+z_{0}^{2}}
$$

The corresponding formula for the tunneling conductance for the $z y$ interface is

$$
\sigma_{\uparrow[\downarrow]}(E)=\frac{\int_{-\pi / 2}^{\pi / 2} \int_{0}^{\frac{\pi}{2}} \bar{\sigma}_{\uparrow[\downarrow]}(E, \phi, \theta) \sin ^{2} \theta \cos \phi d \phi d \theta}{\int_{-\pi / 2}^{\pi / 2} \int_{0}^{\frac{\pi}{2}} \sin ^{2} \theta \cos \phi 2 \sigma_{N} d \phi d \theta}
$$

where the normal-state conductance is given by

$$
\sigma_{N}=\frac{\cos ^{2} \phi \sin ^{2} \theta}{\cos ^{2} \phi \sin ^{2} \theta+z_{0}^{2}} .
$$

The pairing potential is described by a $2 \times 2$ form

$$
\hat{\Delta}_{\alpha, \beta}(\mathbf{k})=\left(\begin{array}{ll}
-d_{x}(\mathbf{k})+i d_{y}(\mathbf{k}) & d_{z}(\mathbf{k}) \\
d_{z}(\mathbf{k}) & d_{x}(\mathbf{k})+i d_{y}(\mathbf{k})
\end{array}\right),
$$

in terms of the $d(\mathbf{k})=\left(d_{x}(\mathbf{k}), d_{y}(\mathbf{k}), d_{z}(\mathbf{k})\right)$ vector.

For $\mathrm{Sr}_{2} \mathrm{RuO}_{4}$ the $d$-vector runs parallel to $z$-axis (i.e., $d(\mathbf{k})=\left(0,0, d_{z}(\mathbf{k})\right)$. The candidate pairing states are given by

a) $d_{z}(\mathbf{k})=\left(k_{x}+i k_{y}\right) \cos \left(c k_{z}\right)$, with $c$ being the lattice constant along the $c$-axis. This state has horizontal lines of nodes at $k_{z}= \pm \frac{\pi}{2 c}$ and breaks the time reversal symmetry.

b) $d_{z}(\mathbf{k})=\left(\sin \left(\frac{a k_{x}}{2}\right)+i \sin \left(\frac{a k_{y}}{2}\right)\right) \cos \left(\frac{c k_{z}}{2}\right)$, with horizontal lines of nodes at $k_{z}= \pm \frac{\pi}{c}$.

c) $d_{z}(\mathbf{k})=\left(k_{x}+i k_{y}\right)^{2} k_{z}$, with horizontal lines of nodes at $k_{z}=0$.

Then we will choose two candidate pairing states corresponding to the $B$-phase of $\mathrm{UPt}_{3}$ (low temperature $T$ and low field $H$ ): a) The unitary planar state with $d(\mathbf{k})=$ $\left(\lambda_{x}(\mathbf{k}), \lambda_{y}(\mathbf{k}), 0\right)$, and b) the non-unitary bipolar state with $d(\mathbf{k})=\left(\lambda_{x}(\mathbf{k}), i \lambda_{y}(\mathbf{k}), 0\right)$, where $\lambda_{x}(\mathbf{k})=k_{z}\left(k_{x}^{2}-k_{y}^{2}\right), \lambda_{y}(\mathbf{k})=k_{z} 2 k_{x} k_{y}$. 
According to the BTK formula the conductance of the junction, $\bar{\sigma}_{\uparrow[\downarrow]}(E)$, for up(down) spin quasiparticles, is expressed in terms of the probability amplitudes $a_{\uparrow[\downarrow]}, b_{\uparrow[\downarrow]}$ as $\mathbb{8}$

$$
\bar{\sigma}_{\uparrow[\downarrow]}(E)=1+\left|a_{\uparrow[\downarrow]}\right|^{2}-\left|b_{\uparrow[\downarrow]}\right|^{2} .
$$

The Andreev and normal reflection amplitudes $a_{\uparrow[\downarrow]}, b_{\uparrow[\downarrow]}$ for the spin-up(-down) quasiparticles are obtained as

$$
\begin{gathered}
a_{\uparrow[\downarrow]}=\frac{4 n_{+}}{4+z_{0}^{2}-z_{0}^{2} n_{+} n_{-} \phi_{-} \phi_{+}^{*}}, \\
b_{\uparrow[\downarrow]}=\frac{-\left(2 i z_{0}+z_{0}^{2}\right)+\left(2 i z_{0}+z_{0}^{2}\right) n_{+} n_{-} \phi_{-} \phi_{+}^{*}}{4+z_{0}^{2}-z_{0}^{2} n_{+} n_{-} \phi_{-} \phi_{+}^{*}},
\end{gathered}
$$

where $z_{0}=\frac{m V}{\hbar^{2} k_{s}}$. The BCS coherence factors are given by

$$
\begin{aligned}
& u_{ \pm}^{2}=\left[1+\sqrt{E^{2}-\left|\Delta_{ \pm}\right|^{2}} / E\right] / 2, \\
& v_{ \pm}^{2}=\left[1-\sqrt{E^{2}-\left|\Delta_{ \pm}\right|^{2}} / E\right] / 2,
\end{aligned}
$$

and $n_{ \pm}=v_{ \pm} / u_{ \pm}$. The internal phase coming from the energy gap is given by $\phi_{ \pm}=$ $\left[\Delta_{ \pm} /\left|\Delta_{ \pm}\right|\right]$, where $\Delta_{+}\left(\Delta_{-}\right)$is the pair potential experienced by the transmitted electron-like (hole-like) quasiparticle.

3. $\mathrm{Sr}_{2} \mathrm{RuO}_{4}$

In Figs. 2-4 we plot the tunneling conductance $\sigma(E)$ as a function of $E / \Delta_{0}$ for various values of $z_{0}$, for the $x y$-interface (a), and $z y$-interface (b), for the superconductor $\mathrm{Sr}_{2} \mathrm{RuO}_{4}$. The pairing symmetry of the superconductor is $\left(k_{x}+i k_{y}\right) \cos \left(c k_{z}\right)$-wave in Fig. 2, $\left(\sin \left(\frac{a k_{x}}{2}\right)+\right.$ $\left.i \sin \left(\frac{a k_{y}}{2}\right)\right) \cos \left(\frac{c k_{z}}{2}\right)$-wave in Fig. 3, $\left(k_{x}+i k_{y}\right)^{2} k_{z}$-wave in Fig. \$.

The conductance peak is formed in the electron tunneling for the $x y$-interface, $z y$ interface when the transmitted quasiparticles experience different sign of the pair potential on the Fermi surface (FS). Also the line shape of the spectra is sensitive to the presence or absence of nodes of the pair potential on the Fermi surface.

For the $\left(k_{x}+i k_{y}\right) \cos \left(c k_{z}\right)$-wave case, for the $x y$-interface the scattering process changes the electron momentum from $(\phi, \pi-\theta)$ to $(\phi, \theta)$ on the FS. However this process conserves 
the sign of the pair potential for $0<\phi<2 \pi$. As a result no peak exists in the conductance spectra, as seen in Fig. 2 (a) for $z_{0}=2.5$. Also the nodes of the pair potential at $k_{z}= \pm \pi / 2 c$ intersect the FS along the $z$-axis and a V-like gap opens in the tunneling spectra as in the case of the $d$-wave superconductor. On the other hand for the $z y$-interface the transmitted quasiparticles feel different sign of the pair potential for $(\phi, \theta)$ and $(\pi-\phi, \theta)$ only at discrete $\phi$-values which explain the residual values of the conductance within the energy gap seen in Fig. 2 (b).

For the $\left(\sin \left(\frac{a k_{x}}{2}\right)+i \sin \left(\frac{a k_{y}}{2}\right)\right) \cos \left(\frac{c k_{z}}{2}\right)$-wave case, and for the $x y$-interface the scattering process in the momentum space connects points of the FS with the same sign as in the $\left(k_{x}+i k_{y}\right) \cos \left(c k_{z}\right)$-wave case. This means that the pair potential does not change sign and

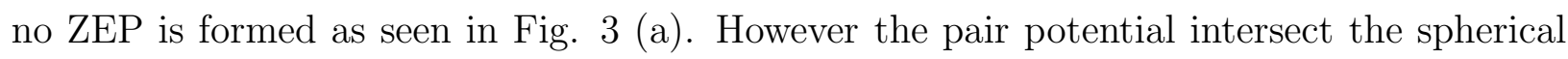
FS at the poles, i.e., at $k_{z}= \pm \pi / c$, forming point like nodes. This explains the logarithmic singularity at $E=0$ at the spectra. The tunneling spectra for the $z y$-interface is enhanced due to the bound states that are formed at discrete values of the quasiparticle angle $\phi$ as seen in Fig. 通 (b).

The situation is opposite in the $\left(k_{x}+i k_{y}\right)^{2} k_{z}$-wave case where the scattering process for $x y$ interface connects points of the FS, i.e., $(\phi, \pi-\theta)$ and $(\phi, \theta)$, with opposite sign. As a

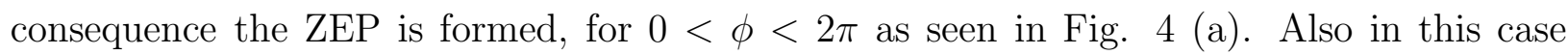
the node of the pair potential at $k_{z}=0$ intersect the FS and the spectra has a V-shaped form as in the $\left(k_{x}+i k_{y}\right) \cos \left(c k_{z}\right)$-wave case. For the $z y$-interface the order parameter has the same $\phi$ dependence as in the $\left(k_{x}+i k_{y}\right) \cos \left(c k_{z}\right)$-wave, and the tunneling spectra for the $z y$-interface is similar.

The conclusion is that the tunneling at the $x y$ interface can be used to distinguish the pairing states with horizontal lines of nodes on the FS. The numerical results presented here are in agreement with recent analytical calculation for the case of the low transparency barrier and triplet pairing states with horizontal lines of nodes [19]. Also only one electron band for $\mathrm{Sr}_{2} \mathrm{RuO}_{4}$ contributes to superconductivity. However it has been proposed recently that the $\gamma$ band is nodeless while $\alpha$ and $\beta$ bands have horizontal lines of nodes [20]. In 
this case all three bands contribute to the pairing state and the actual shape of the spectra depends on the amount of contribution of different bands.

In this paragraph a comparison is made between the pairing states examined here and the corresponding states without the $k_{z}$-dependence. For the $k_{x}+i k_{y}$-wave case, for the $x y$ interface the gap has a U-shaped structure due to the absence of sign change of the order parameter along the $z$-axis. For the $z y$-interface the difference is more pronounced at $z_{0}=0$ where $\sigma(E)$ is constant within the gap for the $k_{x}+i k_{y}$-wave case, while it posses a $\Lambda$ shape structure for the $\left(k_{x}+i k_{y}\right) \cos \left(c k_{z}\right)$-wave.

For the $\sin \left(\frac{a k_{x}}{2}\right)+i \sin \left(\frac{a k_{y}}{2}\right)$-wave, and $x y$-interface the spectra is expected to have the U-shaped line shape, while for the $z y$ interface the spectra should have residual values due to the presence of bound states. However a detailed calculation is needed to account for the actual shape of the spectra.

4. $U P t_{3}$

In Figs. 5-6 we plot the tunneling conductance $\sigma(E)$ as a function of $E / \Delta_{0}$ for various values of $z_{0}$, for the $x y$-interface (a), and $z y$-interface (b) for the superconductor $\mathrm{UPt}_{3}$. The pairing symmetry of the superconductor is the unitary planar in Fig. 5 and the non unitary bipolar in Fig. 6.

The conductance peak is formed in the electron tunneling for the $x y$-interface, $z y$ interface when the transmitted quasiparticles experience different sign of the pair potential on the Fermi surface. Also the line shape of the spectra is sensitive to the presence or absence of nodes of the pair potential on the Fermi surface.

For the planar case, for the $x y$-interface the scattering process changes the electron momentum from $(\phi, \pi-\theta)$ to $(\phi, \theta)$ on the FS. This process changes the sign of the pair potential for $0<\phi<2 \pi$. As a result a peak exists in the conductance spectra, as seen in Fig. 5 (a) for $z_{0}=2.5$. Also the nodes of the pair potential at $k_{z}=0$ intersect the FS along the $z$-axis and a $\mathrm{V}$-like gap opens in the tunneling spectra as in the case of the $d$-wave superconductor. On the other hand for the $z y$-interface the transmitted quasiparticles feel different sign of the pair potential for $(\phi, \theta)$ and $(\pi-\phi, \theta)$ only at discrete $\phi$-values which 
explain the residual values of the conductance within the energy gap seen in Fig. 5 (b).

For the bipolar case, and for the $x y$-interface the scattering process in the momentum space connects points of the FS with different sign as in the planar-wave case. This means that the pair potential changes sign and a ZEP is formed as seen in Fig. 6 (a). The tunneling spectra for the $z y$-interface is enhanced due to the sign change caused by the scattering $(\phi, \theta)$ to $(\pi-\phi, \theta)$ as seen in Fig. 6 (b).

The conclusion is that the tunneling at the $z y$ interface can be used to distinguish the pairing states with horizontal lines of nodes on the FS.

In this paragraph we analyze the pairing state corresponding to the $A$ phase (high $T$, low $H)$ of $\mathrm{UPt}_{3}$, where the secondary order parameter vanishes. The resulting order parameter does not break the time reversal symmetry. For the $x y$ interface the spectra has a ZEP due to the sign change of the order parameter along the $z$-axis as seen in Fig. 7(a). Also the line shape of the spectra is $\mathrm{V}$-like because the nodes of the pair potential at $k_{z}=0$ intersect the FS. For the $z y$-interface the scattering process from $(\phi, \theta)$ to $(\pi-\phi, \theta)$ does not change the sign of the pair potential and no ZEP occurs as seen in Fig. 7(b). However the line nodes which run parallel to $k_{z}$ intersect the FS and the spectra is $V$-shaped.

\section{Experimental relevance}

In this section a comparison is made with existing tunneling experiments on $\mathrm{Sr}_{2} \mathrm{RuO}_{4}$ and $\mathrm{UPt}_{3}$. Tunneling experiments that have been performed on cleaved $c$-axis junctions of Ru-embedded $\mathrm{Sr}_{2} \mathrm{RuO}_{4}$ show a bell shaped spectrum with a sharp peak at zero bias for the 1.4- $\mathrm{T}$ phase and a sharp ZEP for the $3-\mathrm{K}$ phase 21]. The spectra for the $1.4-\mathrm{K}$ phase is similar to the board ZEP observed in $\mathrm{Ru}$-free $\mathrm{Sr}_{2} \mathrm{RuO}_{4}$ via point contact spectroscopy [22] although their experiments actually measure the tunneling resistance. This type of spectra for the 1.4-K phase is consistent with nodeless Eu pairing state where the broadening of the ZEP is due to the presence of Andreev bound states. The sharp peak seen in the 3-K phase is an indication of a pairing state with horizontal lines of nodes. It has been suggested that a phase transition occurs where the $3-\mathrm{K}$ phase with line node transforms to a nodeless Eu state close to the bulk $T_{c}$ [23]. In the experiments of Mao et al. [21] the tunneling direction 
is along the $c$-axis. However the experimentalists believe that the Andreev reflection takes place in the in-plane direction due to the Ru inclusions. Then the pairing state that fits well the experimental data for the $z y$ interface is $d_{z}(\mathbf{k})=\left(k_{x}+i k_{y}\right) \cos \left(c k_{z}\right)$ as seen in Fig. 2(b), rather than $d_{z}(\mathbf{k})=\left(\sin \left(\frac{a k_{x}}{2}\right)+i \sin \left(\frac{a k_{y}}{2}\right)\right) \cos \left(\frac{c k_{z}}{2}\right)$ and $d_{z}(\mathbf{k})=\left(k_{x}+i k_{y}\right)^{2} k_{z}$, as seen in Figs. (3)(b) and G(b). On the other hand if the Andreev reflection occurs in the $c$-axis direction then the pairing state which fits more precisly the experimental data is $d_{z}(\mathbf{k})=\left(k_{x}+i k_{y}\right)^{2} k_{z}$ which shows a clear ZEP for the $x y$-interface, for the low transparency barrier as seen in Fig. 四(a).

Point contact spectroscopy for heavy fermion superconductors $\mathrm{UPt}_{3}$ has been performed where distinct minima in the differential resistance versus voltage have been observed for current flow parallel to the $c$-axis, and only very weak structures-if at all- have been observed for current flow within the basal plane [24]. Their observation is consistent with the calculated tunneling conductance for the unitary planar state seen in Fig. 5(a) and 5(b), for the tunneling conductance along the $c$-axis and $a$-axis respectively. Moreover measurements of the differential conductivity of $\mathrm{UBe}_{13}-\mathrm{Au}$ contacts, where the $\mathrm{UBe}_{13}$ is in polycrystalline form, reveal the existence of low-energy Andreev surface bound states, which are identified by the presence of ZEP and are consistent with an order parameter with nontrivial symmetry of the energy gap [25].

\section{Conclusions}

We calculated the tunneling conductance in normal-metal/insulator/triplet superconductor with horizontal lines of nodes, junction using the BTK formalism. We assumed possible pairing potentials for the superconductor which break the time reversal symmetry. For the $\mathrm{Sr}_{2} \mathrm{RuO}_{4}$ the tunneling at the $x y$ interface can be used to distinguish the pairing states with horizontal lines of nodes from the ZEP that is formed when the pair potential changes its sign on the FS during the scattering process. Also for the $x y$ interface the line shape of the spectra is V-like due to the presence of nodes of the pair potential along the $z$ axis at the FS, while for the $z y$ interface the tunneling conductance has residual values due to the formation of bound states at discrete values of the angle $\phi$. 
For the $\mathrm{UPt}_{3}$ for the tunneling along the $z$-axis a $\mathrm{ZEP}$ is formed for the pairing states we examined, while the spectra along the $x$-axis has residual values or develops a ZEP depending on the details of the pairing state. In each case the observation of a ZEP in the tunneling experiments is consistent with an order parameter with nontrivial symmetry of the energy gap. 


\section{REFERENCES}

[1] Y. Maeno, H. Hashimoto, K. Yoshida, S. Nishizaki, T. Fujita, J.G. Bednorz, and F. Lichtenberg, Nature (London) 372, 532 (1994).

[2] K. Ishida, H. Mukuda, Y. Kitaoka, K. Asayama, Z.Q. Mao, Y. Mori, and Y. Maeno, Nature 396, 658 (1998).

[3] G.M. Luke, Y. Fukamoto, K.M. Kojima, M.L. Larkin, J. Merrin, B. Nachumi, Y.J. Uemura, Y. Maeno, Z.Q. Mao, Y. Mori, H. Nakamura, and M. Sigrist, Nature (London) 394, 558 (1998).

[4] K. Ishida, Y. Kitaoka, K. Asayama, S. Ikeda, S. Nishizaki, Y. Maeno, K. Yoshida, and T. Fujita, Phys. Rev. B 56, 505 (1997).

[5] S. Nishizaki, Y. Maeno, and Z. Mao, J. Phys. Soc. Jpn. 69, 572 (2000).

[6] H. Tou, Y. Kitaoka, K. Ishida, K. Asayama, N. Kimura, Y. Onuki, E. Yamamoto, Y. Haga, and K. Maezawa, Phys. Rev. Lett. 80, 3129 (1998).

[7] G.M. Luke, A. Keren, L.P. Le, Y.J. Uemura, D.A. Bonn, L. Taillefer, and J.D. Garrett, Phys. Rev. Lett. 71, 1446 (1993).

[8] G.E. Blonder, M. Tinkham, and T.M. Klapwijk, Phys. Rev. B 25, 4515 (1982).

[9] A.F. Andreev, Soviet Phys. JETP 19, 1228 (1964).

[10] M. Covington, M. Aprili, E. Paraoanu, L.H. Green, F. Xu, J. Zhu, and C.A. Mirkin, Phys. Rev. Lett. 79, 277 (1997).

[11] N. Stefanakis, J. Phys.: Condens. Matter 13, 1265 (2001).

[12] N. Stefanakis, J. Phys.: Condens. Matter 13, 3643 (2001).

[13] N. Stefanakis, cond-mat/0109498.

[14] M. Yamashiro, Y. Tanaka, and S. Kashiwaya, Phys. Rev. B 56, 7847 (1997). 
[15] M. Yamashiro, Y. Tanaka, Y. Tanuma, and S. Kashiwaya, J. Phys. Soc. Jpn. 67, 3224 (1998).

[16] Y. Hasegawa, K. Machida, and M. Ozaki, J. Phys. Soc. Jpn. 69, 336 (2000).

[17] H. Won and K. Maki, Europhys. Lett. 52, 427 (2000).

[18] K. Machida, T. Nishira, and T. Ohmi, J. Phys. Soc. Jpn. 68, 3364 (1999).

[19] K. Sengupta, H.-J. Kwon, and V.M. Yakovenko, cond-mat/0106198.

[20] M.E. Zhitomirsky and T.M. Rice, Phys. Rev. Lett. 87, 057001 (2001).

[21] Z. Q. Mao, K. D. Nelson, R. Jin, Y. Liu, and Y. Maeno, Phys. Rev. Lett. 87, 037003 (2001).

[22] F. Laube, G. Goll, H. v. Lohneysen, M. Fogelstrm, and F. Lichtenberg Phys. Rev. Lett. 84, 1595 (2000).

[23] M. Sigrist, and H. Monien, J. Phys. Soc. Jpn. 70, 2409 (2001).

[24] G. Goll, H. v. Lohneysen, I. K. Yanson, and L. Taillefer, Phys. Rev. Lett. 70, 2008 (1993).

[25] Ch. Walti, H. R. Ott, Z. Fisk, and J. L. Smith, Phys. Rev. Lett. 84, 5616 (2000). 
(a)

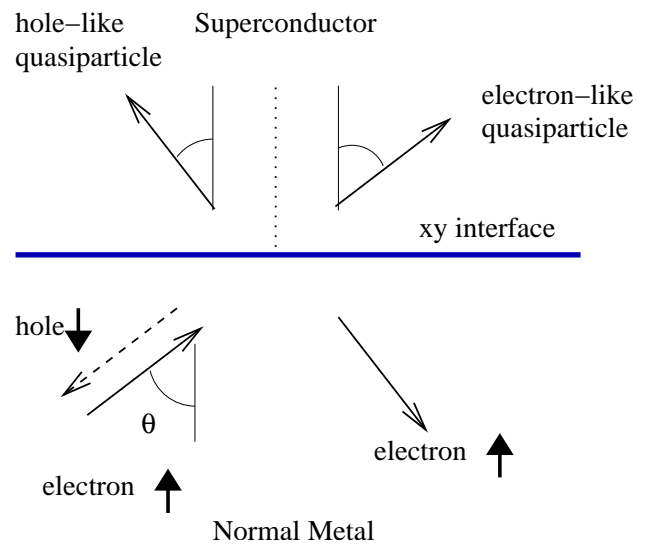

(b)

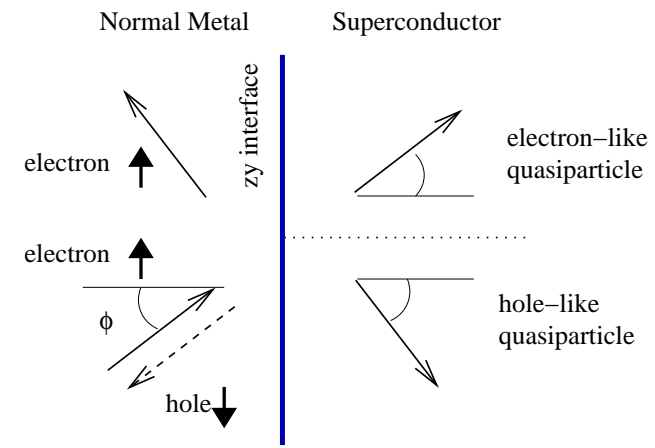

FIG. 1. The figure illustrate the transmission and reflection processes of the quasiparticle at the interface of the junction with (a) $x y$ plane interface, (b) $z y$ plane interface. 


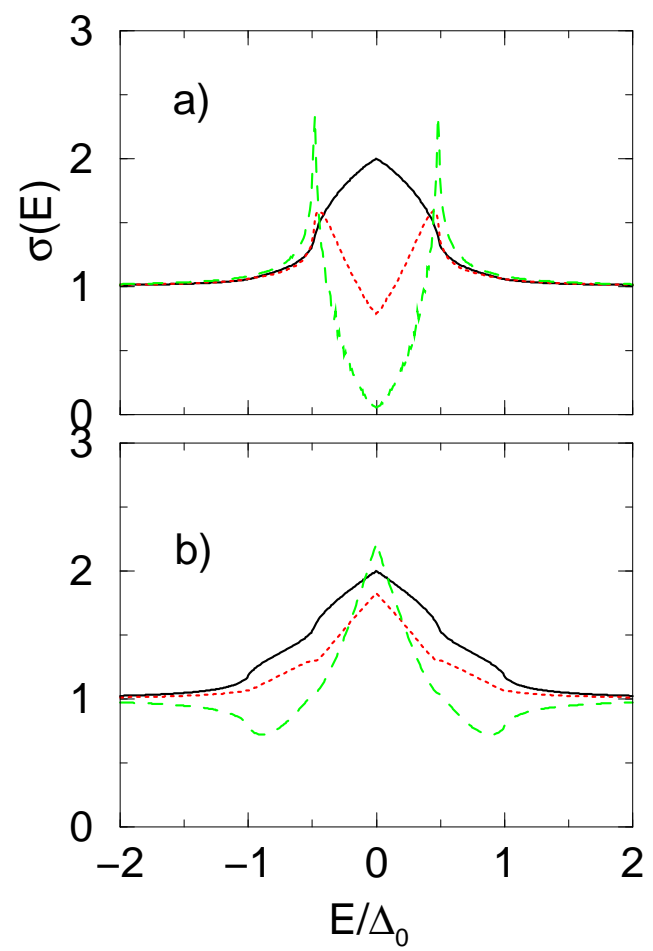

FIG. 2. Normalized tunneling conductance $\sigma(E)$ as a function of $E / \Delta_{0}$ for $z_{0}=0$ (solid line), $z_{0}=0.5$ (dotted line), $z_{0}=2.5$ (dashed line), for the superconductor $\mathrm{Sr}_{2} \mathrm{RuO}_{4}$. In (a) the interface is perpendicular to the $z$-axis, and in (b) the interface is perpendicular to the $x$-axis. The pairing symmetry of the superconductor is $\left(k_{x}+i k_{y}\right) \cos \left(c k_{z}\right)$-wave. 


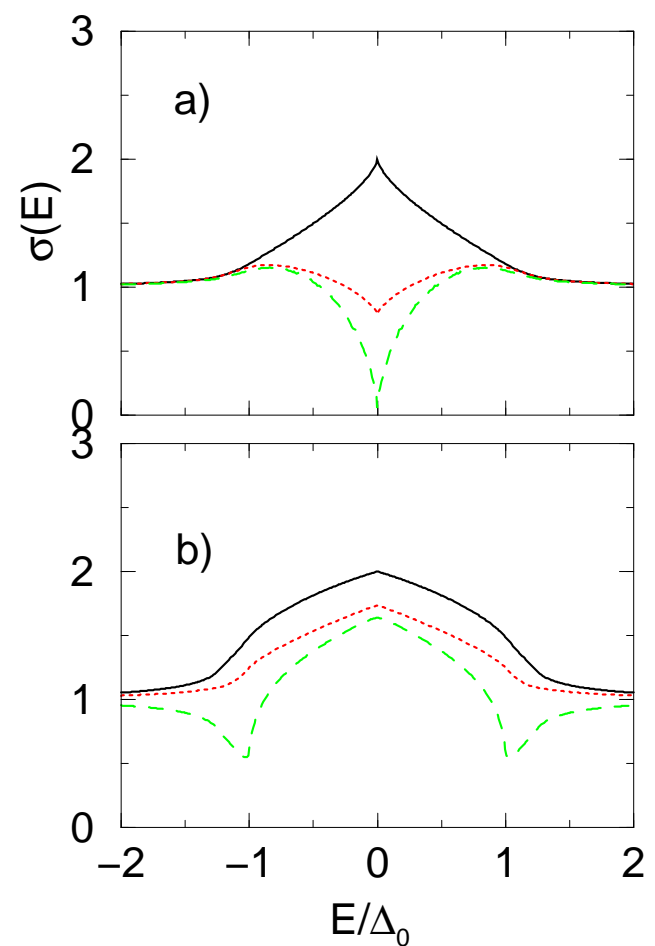

FIG. 3. The same as in Fig. 3. The pairing symmetry of the superconductor is $\left(\sin \left(\frac{a k_{x}}{2}\right)+i \sin \left(\frac{a k_{y}}{2}\right)\right) \cos \left(\frac{c k_{z}}{2}\right)$-wave.

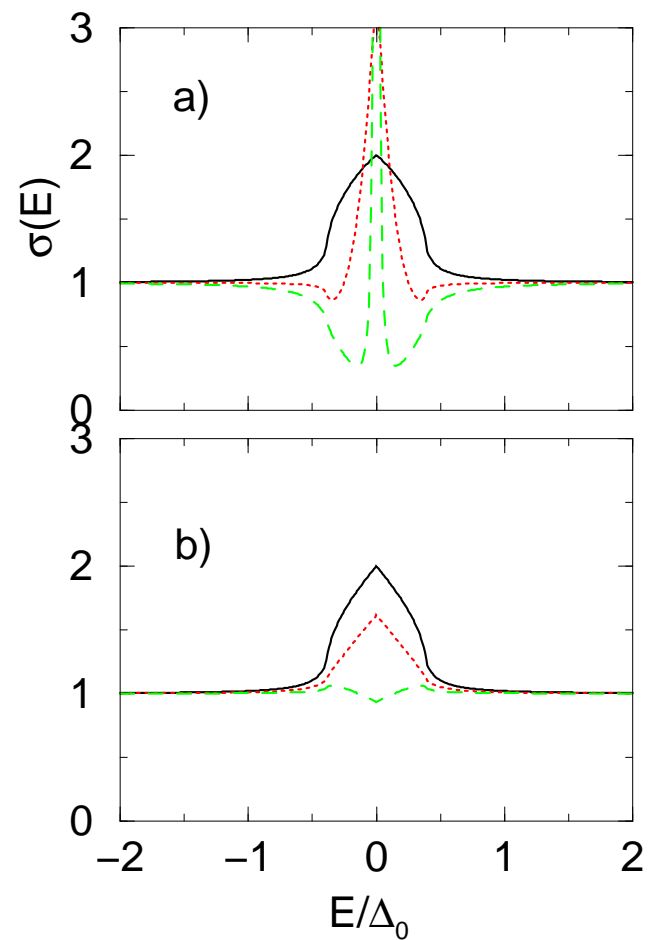

FIG. 4. The same as in Fig. 3. The pairing symmetry of the superconductor is $\left(k_{x}+i k_{y}\right)^{2} k_{z}$-wave. 


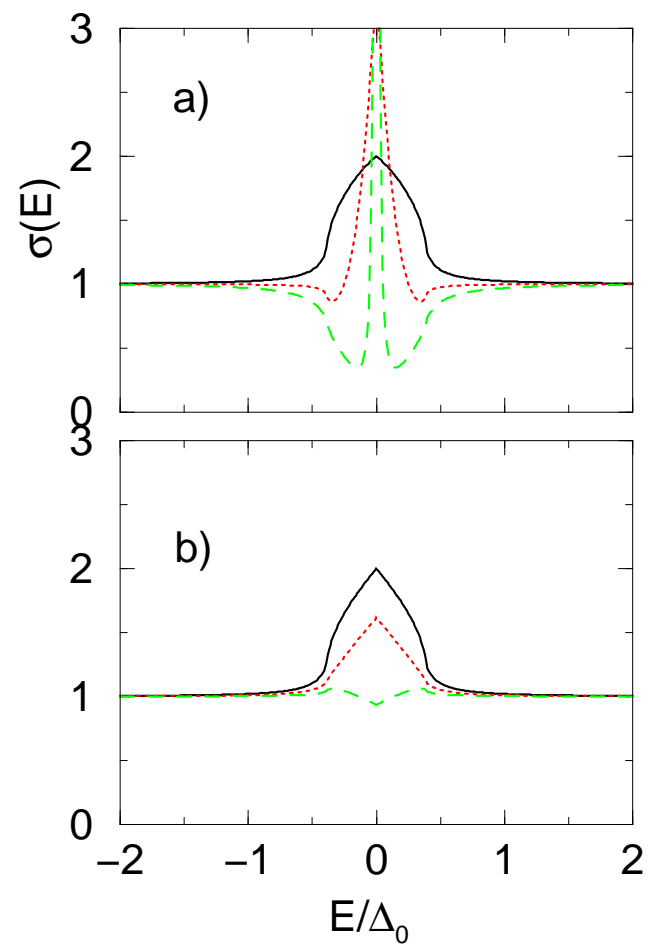

FIG. 5. Normalized tunneling conductance $\sigma(E)$ as a function of $E / \Delta_{0}$ for $z_{0}=0$ (solid line), $z_{0}=0.5$ (dotted line), $z_{0}=2.5$ (dashed line), for the superconductor $\mathrm{UPt}_{3}$. The pairing symmetry of the superconductor is planar.

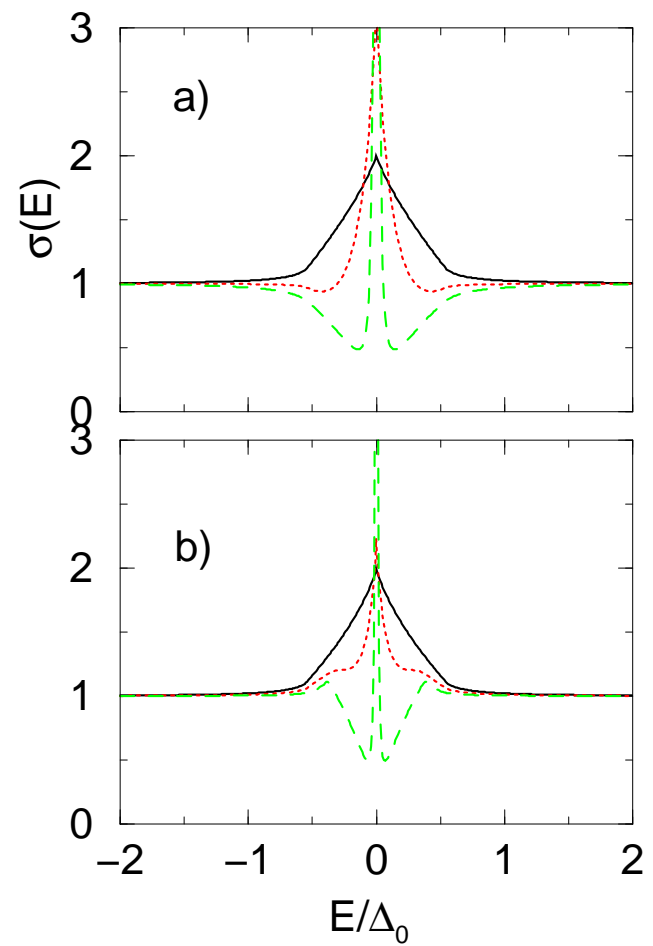

FIG. 6. The same as in Fig. 3. The pairing symmetry of the superconductor is bipolar. 


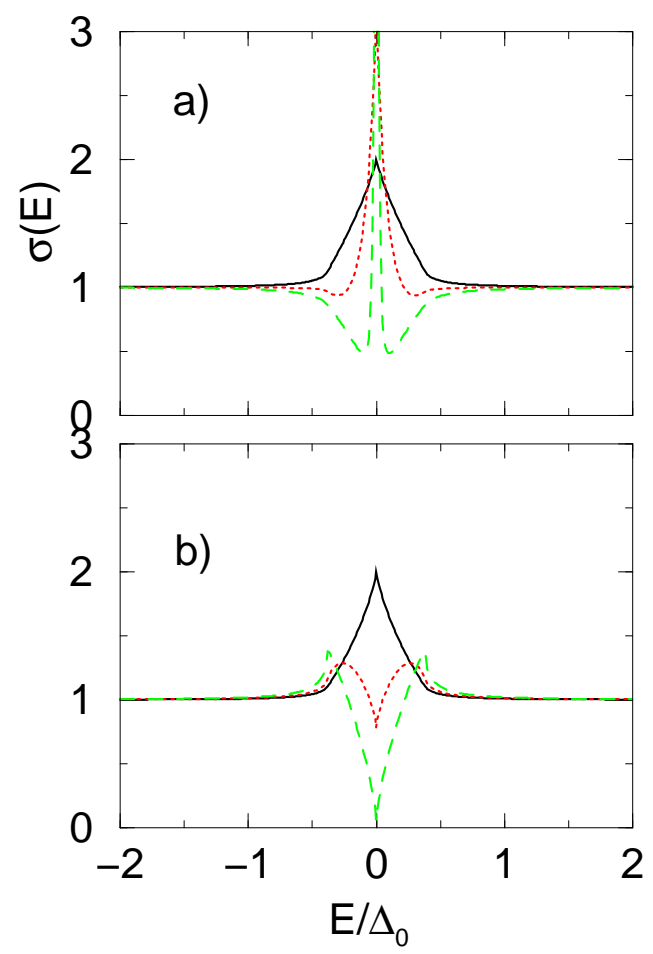

FIG. 7. The same as in Fig. 3. The pairing symmetry describes the $A$ phase of $\mathrm{UPt}_{3}$ where the secondary component of the order parameter vanishes. 